\title{
Tandem Radical Fragmentation/Cyclization of Guanidinylated Monosaccharides Grants Access to Medium-Sized Polyhydroxylated Heterocycles
}

\author{
Andrés G. Santana, ${ }^{* \dagger}$ Concepción C. González* \\ Instituto de Productos Naturales y Agrobiología del C.S.I.C., Avenida Astrofísico Francisco Sánchez 3, \\ 38206 La Laguna, Tenerife, Spain.
}

\begin{abstract}
The fragmentation of anomeric alkoxyl radicals (ARF) and the subsequent cyclization promoted by hypervalent iodine provides an excellent method for the synthesis of guanidino-sugars. The methodology described herein is one of the few existing general methodologies for the formation of medium-sized exo- and endo-guanidine-containing heterocycles presenting a high degree of oxygenation in their structure
\end{abstract}

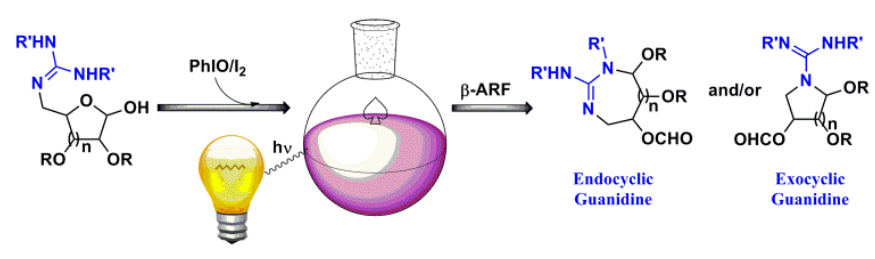

The guanidine functional group is a structural motif frequently found in marine natural products that provides them with potent biological activities. ${ }^{1}$ In fact, the interest in this type of compounds has increased dramatically in recent years, as it has been shown that the incorporation of this moiety into a given molecule can lead to a wide diversity of applications, ${ }^{2}$ among which the development of new drugs,${ }^{3}$ organocatalysts ${ }^{4}$ and even new molecular materials ${ }^{5}$ can be highlighted. The most remarkable natural compounds within this group are those presenting a cyclic guanidinium, such as saxitoxin $(\mathbf{I}){ }^{6}$ tetrodotoxin $(\mathbf{I I})^{7}$ or crambescin A $(\text { III })^{8}$ (Figure 1$)$. Due to the complexity of their structures, these natural products have posed a synthetic challenge for organic chemists, which in turn has led to the development of numerous synthetic methodologies, especially in the synthesis of 5and 6-membered cyclic guanidines. ${ }^{9}$ Natural products containing higher-order cyclic guanidines are, to the best of our knowledge, much scarcer and in fact only monanchorin (IV),${ }^{10}$ which contains a bridged 7-membered ring, is reported in the literature. For that reason, synthetic processes aimed at obtaining these structures are much less frequent; however, it is worth mentioning that a 9-membered cyclic guanidine $(\mathbf{V})^{11}$ has been reported as an intermediate in the synthesis of saxitoxine, and that a few serendipitous 7 -membered guanidines (VI) ${ }^{12}$ have also been described as inhibitors of HIV1 proteases (Figure 1).

In our research group, we have been interested in the search for new potentially biologically active compounds employing as a key reaction the $\beta$-fragmentation of alkoxyl radicals ( $\beta$-ARF) derived from carbohydrates. ${ }^{13}$ 


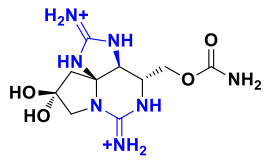

(+) Saxitoxin (I)

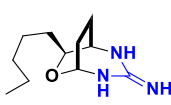

Monanchorin (IV)

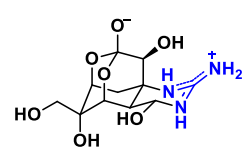

Tetrodotoxin (II)

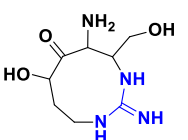

(V)

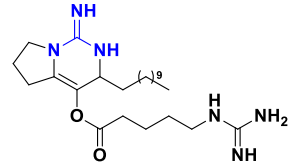

Crambescin A (III)

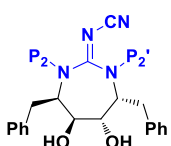

(VI)

Figure 1. Illustrative examples of natural and synthetic cyclic guanidinium-containing compounds.

In our research group, we have been interested in the search for new potentially biologically active compounds employing as a key reaction the $\beta$-fragmentation of alkoxyl radicals ( $\beta$-ARF) derived from carbohydrates. ${ }^{13}$ As part of the general efforts aimed at the preparation of novel nitrogenous compounds as potential glycosidase inhibitors, ${ }^{14}$ we have focused our attention on the study of different nitrogen-containing nucleophiles leading to novel polyhydroxylated heterocycles of interest not only because of their structure, but also for their putative activities. ${ }^{15}$ In this regard, we have been able to synthesize a battery of imino-sugars ${ }^{15 a}$ and iminoketoses ${ }^{15 b}$ using protected amines, as well as tetrazolo-sugars ${ }^{15 \mathrm{c}}$ and benzimidazolo-sugars, ${ }^{15 \mathrm{~d}}$ for which the corresponding heterocycles were used as the nucleophilic counterpart, for the first time, in such cyclization reactions. Considering the current relevance and usefulness of the guanidine functional group, and having recently developed a straightforward method for obtaining guanidines from azides in a one-pot fashion, ${ }^{16}$ we decided to carry out the synthesis of cyclic guanidines derived from carbohydrates employing an analogous methodology to that previously reported.

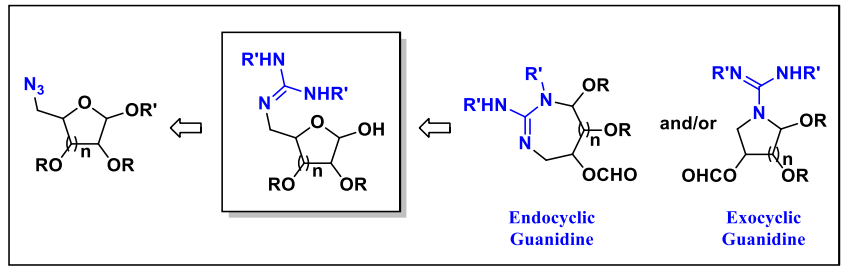

Scheme 1. Proposed retrosynthetic analysis.

Nevertheless, this time the internal nucleophile, a conveniently positioned Boc-protected guanidine group, would be responsible for the intramolecular cyclization, rather than an amido or an $N$-heterocyclic group. Another difference with earlier works arises from the bidentate character of the guanidine group as a nucleophile, which opens the possibility of differentiating between the two non-equivalent nitrogen atoms, thus turning the proposed methodology more versatile, as products of different sizes (endocyclic $v s$ exocyclic guanidine) ${ }^{17}$ could be generated (Scheme 1). 
Table 1. Synthesis of hemiacetals 12-17. ${ }^{\mathrm{a}}$

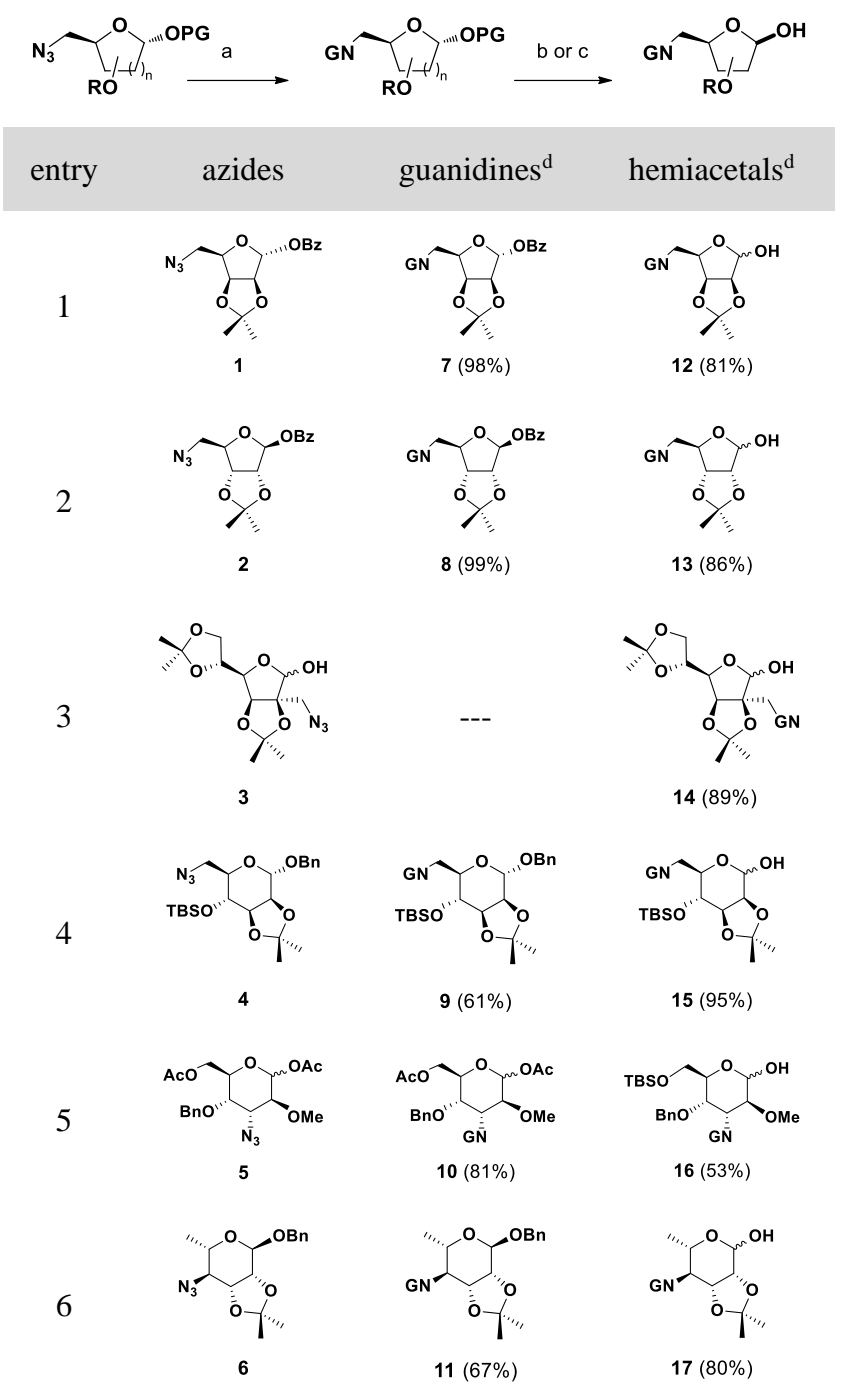

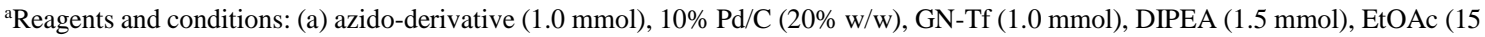
$\mathrm{mL} / \mathrm{mmol}$ ), under $\mathrm{H}_{2}$ atmosphere (balloon), overnight; (b) MeONa, MeOH, rt; (c) $\mathrm{Pd}(\mathrm{OH})_{2} / \mathrm{C}(30 \%), \mathrm{H}_{2}$ (1 atm), EtOAc, rt; (d) Isolated yields.

This unprecedented procedure, which could help elucidate the mechanistic preference governing the intramolecular cyclization, would concomitantly allow the preparation of cyclic polyhydroxylated guanidines of higher order. ${ }^{18}$ With this idea in mind, we decided to prepare a series of carbohydrate models where the guanidine group was located in different positions, thus allowing us to study the scope in reactivity of both primary and secondary guanidines. The synthetic sequence carried out is described in Table 1. As starting substrates, we resorted to different azido derivatives prepared from carbohydrates in a few steps, either in the furanose or the pyranose form (1-6). ${ }^{16}$ The hydrogenation of the azido group in the presence of a tertiary amine, and the subsequent in situ protection of the resulting amine with Goodman's guanidinylating reagent ( $N, N^{\prime}$-diBoc- $N$ triflyl-guanidine, GN-Tf), ${ }^{19}$ generated the corresponding guanidinylated compound in good to excellent yields. Next, the selective deprotection of the anomeric alcohol, employing either Zemplén transesterification conditions or a hydrogenolysis promoted by Pearlman's catalyst, furnished the desired precursor hemiacetals for the $\beta$ fragmentation/cyclization reaction (12-17). 
In order to test our hypothesis, and to maximize the production of cyclic compounds, the radical fragmentation reaction was carried out using the iodosylbenzene/iodine $\left(\mathrm{PhIO} / \mathrm{I}_{2}\right)$ system in dry dichloromethane, so that the disfavored medium-sized cyclizations would not have to compete with the acetate ions the more common diacetoxy-iodobenzene reagent would liberate in the reaction medium. To our delight, the photolysis of the Dlyxo derivative 12 under visible light showed a total consumption of the starting material in $3.5 \mathrm{~h}$, to produce a quasi-equimolecular mixture of three different products, in an overall yield of $83 \%$ (table 2, entry 1). Given the bidentate nature of the internal nucleophile mentioned earlier, we were anticipating the formation of two compounds: one exocyclic 5-membered and one endocyclic 7-membered guanidine, since both exo-trig cyclizations would be favored according to Baldwin's rules. The key to the formation of the third product lay in the possibility of 7-membered rings to fuse in a cis or trans fashion to the dioxolane ring. The assignment of the different structures was not straightforward, due to the similarities between these regioisomers, and a combination of different $1 \mathrm{D}$ and $2 \mathrm{D}-\mathrm{NMR}$ experiments, including nOe and ${ }^{1} \mathrm{H} /{ }^{15} \mathrm{~N}-\mathrm{HMBC}$ were necessary to fully characterize all these structures. Additionally, these products showed a conformational equilibrium in solution, probably triggered by a slow rotation of the carbamates, whose dependence with temperature was also studied (see Supp. Info. details). Encouraged by these promising results, the same protocol was applied to the remaining hemiacetals 13-17 (Table 2).

Similarly, when compound $\mathbf{1 3}$ derived from D-ribose and structurally related to compound $\mathbf{1 2}$ was irradiated, again three products were isolated under the same conditions, albeit with a lower yield of the exocyclic guanidine 21 (5\%) in favor of the larger sized rings. The fact that the major azepanic compounds 22 and 23 slowly rearranged into the pyrrolidine derivative 21 upon standing in $\mathrm{CDCl}_{3}$ solution indicates both that the 5-membered exocyclic guanidine is indeed more stable, and therefore that the $\beta$-ARF/cyclization reaction takes place under kinetic control, thus favoring the formation of the more unstable, larger sized heterocycles. For the characterization of the corresponding structures, a similar set of NMR experiments to those employed in the previous case was used for this model. The photolysis of hemiacetal $\mathbf{1 4}$ was particularly remarkable, as it produced in only 30 minutes an exclusive spirocyclic compound in $71 \%$ yield (Table 2, entry 3 ). Interestingly, NMR analysis of this new structure revealed the cyclization had occurred with a net retention of the configuration at former position $\mathrm{C}-2$, implying that no rehybridation of the electrophilic carbon took place after the oxidation of the corresponding $C$-radical, which is also in line with a kinetic control of the second step, or that the nucleophilic attack on such oxocarbenium-like intermediate occurs preferentially on one side of the $\mathrm{sp}^{2}$ hybridized carbon.

Table 2. Synthesis of cyclic guanidines 18-29. ${ }^{a}$

\begin{tabular}{|c|c|c|c|}
\hline $\begin{array}{l}\text { starting } \\
\text { material }\end{array}$ & $\mathrm{t}(\mathrm{h})$ & $\begin{array}{l}\text { exocyclic } \\
\text { guanidine } \\
(\text { yield \%) }\end{array}$ & $\begin{array}{l}\text { endocyclic } \\
\text { guanidine/s } \\
\text { (yield \%) }\end{array}$ \\
\hline 12 & 3.5 & $\begin{array}{l}\text { носо } \\
18(27 \%)\end{array}$ & 19(23\%) \\
\hline 13 & 1.0 & 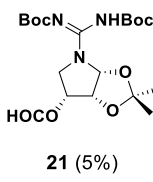 & 22 \\
\hline
\end{tabular}




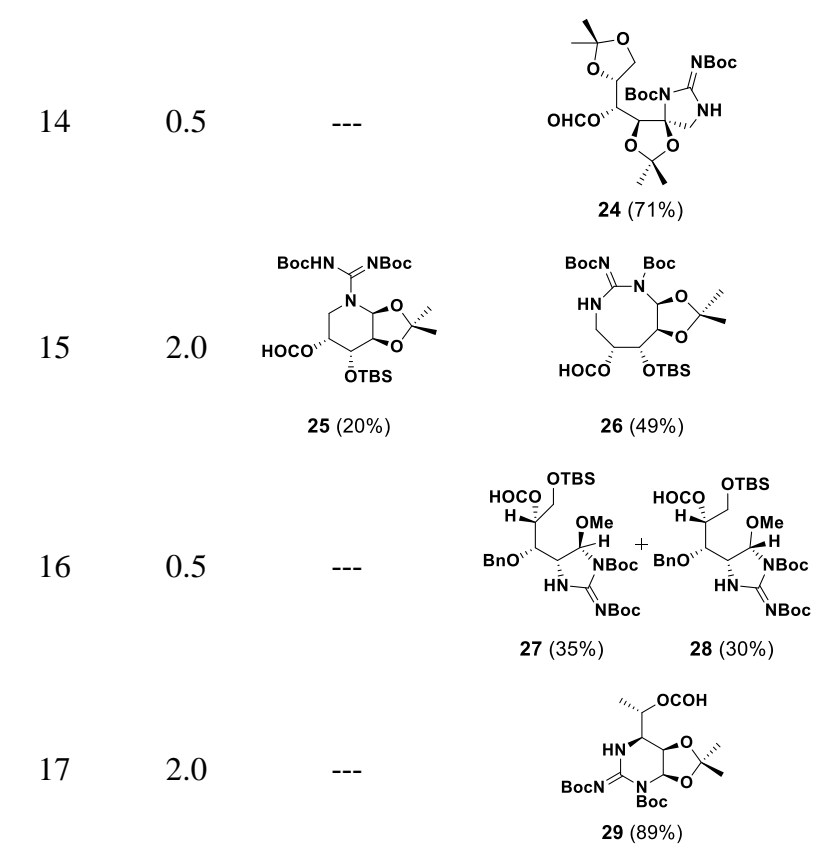

${ }^{\mathrm{a} C}$ Conditions and reagents per mmol of hemiacetal: PhIO (2.2 mmol), $\mathrm{I}_{2}(1.2 \mathrm{mmol})$, DCM ( $\left.25 \mathrm{~mL} / \mathrm{mmol}\right)$, hv, r.t.

To further dissect the scope of this reaction, the pyranose model 15 was prepared, as it could collapse in a 6membered (exo) or an 8-membered ring (endo). When treated with $\mathrm{PhIO} / \mathrm{I}_{2}$, two products, identified as the piperidine 25 and the 1,3-diazocine 26 were obtained in a 1:2.5 ratio and with an overall yield of $69 \%$. Surprisingly, this time no products corresponding to the trans-closure was purified, although other minor byproducts were detected but could not be isolated. As in previous cases, the predominant product is the kinetic 8-membered cyclic product, which is an outstanding finding given the difficulty inherent to these particular cycles.

Interestingly, the photolysis of the secondary guanidine $\mathbf{1 6}$ led quickly and with good overall performance to an almost equimolecular mixture of $\mathbf{2 7}$ and 28, both 5-membered epimeric rings at the closing position, which however were easily separated by rotary chromatography. The fact that only the endocyclic guanidines were obtained this time underscores the preferential formation of bigger sized rings. However, the almost null stereodiscrimination exhibited by the nucleophilic attack does suggest that, contrary to the photolysis of model $\mathbf{1 4}$, in this case a transient $\mathrm{sp}^{2}$-hybridized oxocarbenium-like intermediate must have formed upon oxidation, thus rendering compounds $\mathbf{2 7}$ and $\mathbf{2 8}$ almost in equal rates.

Finally, the photolysis of model system 17 also proceeded smoothly and with good yield to afford pyrimidine derivative 29. In this case, only one endocyclic guanidine was isolated, as the rigidity imposed by 6-membered rings seems incompatible with a trans fusion. Moreover, given that 4-exo-trig-cyclizations are not as favored as the corresponding 6-exo-trig alternatives also rules out the formation of the exocyclic azetidine. This particular reaction was carried out in multigram scale with no apparent loss of yield, but with a slight increase in reaction time.

A plausible mechanism, by which the tandem $\beta$-ARF/cyclization reaction proceeds, illustrated by the transformation of D-lyxo-derivative 12, is depicted in Scheme 2. Initially, an anomeric alkoxyl hipoiodite is generated by the action of the $\mathrm{PhIO} / \mathrm{I}_{2}$ system on the anomeric alcohol. This labile group rapidly experiences a visible light promoted homolytic scission to generate the corresponding alkoxyl radical, which in turn undergoes 
a $\beta$-framentation of the $\mathrm{C} 1-\mathrm{C} 2$ bond, thus generating a formate group from the former acetalic center and a new $C$-centered radical at position C-2. Under the mild conditions inherent to this reaction, and because of the electron-donating nature of the substituent at this position, this radical can now be oxidized to give rise to an oxocarbenium ion-like intermediate, which is eventually trapped by the internal nucleophile to render the desired cyclic guanidines. According to our results, this last nucleophilic step occurs under kinetic control, thus favoring the formation of larger sized rings over that of smaller, more stable compounds. This could be due to a higher nucleophilic character of the NHBoc nitrogen atoms over the internal NH-nitrogen, attributable to either electronic or steric factors.

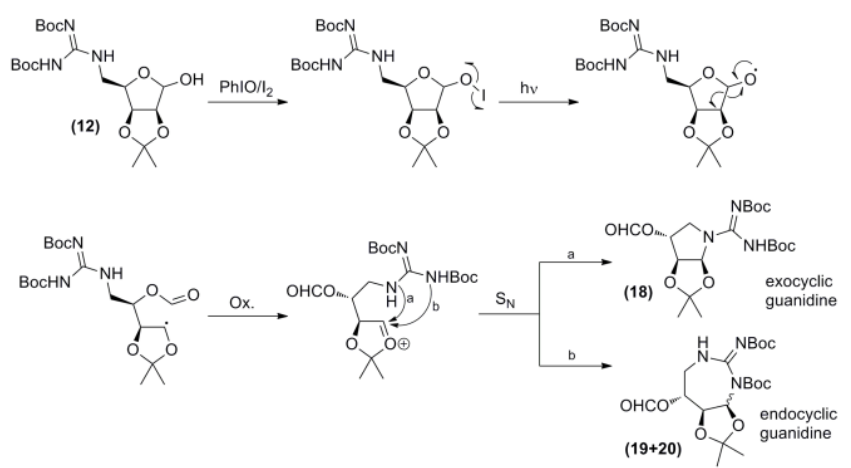

Scheme 2. Plausible reaction mechanism for the synthesis of cyclic guanidines $\mathbf{1 8}, \mathbf{1 9}$ and $\mathbf{2 0}$.

Notwithstanding, the successful preparation of these medium-sized guanidines demonstrates the robust versatility and novelty of the $\beta$-ARF/cyclization protocol, enabling the synthesis of polyhydroxylated nitrogen-containing cyclic structures otherwise difficult to access by any reported method to date. It should also be noted that the methodology described herein is one of the few general existing methods for the formation of exo- and endoguanidine sugars presenting oxidation at the pseudo-anomeric position, which gives these compounds a greater degree of sugar character and could potentially permit subsequent derivatization through this hemiaminal-like center. $^{20}$

In our opinion, the proposed methodology can be readily implemented in the synthesis of numerous guanidinecontaining products, given the mild oxidative conditions inherent to this protocol and the high degree of tolerance for different functional and protecting groups, as evidenced by the heavily functionalized nature of our model saccharidic scaffolds.

\section{ASSOCIATED CONTENT}

\section{Supporting Information}

Synthetic Procedures, NMR characterization and mass spectrometry. The Supporting Information is available free of charge on the ACS Publications website.

\section{AUTHOR INFORMATION}

Corresponding Author

* andres.g.santana@csic.es; $\underline{\text { ccgm @ipna.csic.es }}$

Present Address

$†$ Instituto de Química Orgánica General (IQOG) - CSIC. Calle Juan de la Cierva 3. Madrid (28006), Spain. 


\section{ACKNOWLEDGMENT}

This research study was supported by Mineco/Feder (SAF-2013-48399-R). AGS thanks the I3P-CSIC Program for a fellowship and MINECO for a JdC-incorporación contract.

\section{REFERENCES}

1.- (a).- Berlinck, R. G. S.; Bertonha, A. F.; Takai, M.; Rodriguez, J. P. G. Nat. Prod. Rep. 2017, 34, 1264-1301. (b) Ma, Y.; De, S.; Chen, C. Tetrahedron 2015, 71, 1145-1173. (c) Zárate, S. G.; Santana, A. G.; Bastida, A., Revuelta, J. Curr. Org. Chem. 2014, 18-21, 2711-2749.

2.- Alonso-Moreno, C.; Antiñolo, A.; Carrillo-Hermosilla, F.; Otero, A. Chem. Soc. Rev. 2014, 43, 3406-3425.

3.- (a) Perry, D. L. Jr.; Roberts, B. F.; Debevec, G.; Michaels, H. A.; Chajrabarti, D.; Nefzi, A. Molecules 2019, 24, 1100; doi:10.3390/molecules24061100. (b) Duca, G.; Aricu, A.; Kuchkova, K.; Secara, E.; Barba, A.; Dragalin, I.; Ungur, N.; Spengler, G. Nat. Prod. Res. 2019, 33:21, 3052-3056. (c) Kapp, T. G.; Fottner, M.; Maltsev, O. V.; Kessler, H. Angew. Chem. Int. Ed. 2016, 55, 1540-1543.

4.- (a) Chou, H.-C.; Leow, D.; Tan, C.-H. Chem. Asian. J. 2019, 14, 3803-3822. (b) Hosoya, K.; Odagi, M.; Nagasawa, K. Tetrahedron Lett. 2018, 59, 687-696. (c) Wild, U.; Schön, F.; Himmel, H.-J. Angew. Chem. Int. Ed. 2017, 56, 16410-16413.

5.- (a) Menghui, X.; Xinghuia, J.; Jianhuaa, Z.; Bingcheng, H. Quim. Nova 2019, 42 (2), 181-191. (b) Ji, J.; Zhu; W.; Li, J.; Wang, P.; Liang, Y.; Zhang, W.; Yin, X.; Wu, B., Li, G. ACS Appl. Mater. Interfaces 2017, 9 (22), 19124-19134. (c) Kudo, M.; Tanatani, A. New J. Chem. 2015, 39, 3190-3196.

6.- Tsuchiya, S.; Cho, Y.; Yoshioka, R.; Konoki, K.; Nagasawa, K.; Oshima Y.; Yotsu-Yamashita, M. Angew. Chem. Int. Ed. 2017, 56, 5327-5331, and references cited therein.

7.- Baidilov, D.; Rycek, L.; Trant, J. F.; Froese, J.; Murphy, B.; Hudlicky, T. Angew. Chem. Int. Ed. 2018, 57, 10994-10998 and references cited therein.

8.- (a) Nakazaki, A.; Nakane, Y.; Ishikawa, Y.; Yotsu-Yamashita, M.; Nishikawa, T. Org. Biomol. Chem. 2016, 14, 53045309. (b) El-Demerdash, A.; Moriou, C.; Martin, M.-T.; Rodrigues-Stien, A. S.; Petek, S.; Demoy-Schneider, M.; Hall, K.; Hooper, J. N. A.; Debitus, C.; Al-Mourabit, A. J. Nat. Prod. 2016, 79, 1929-1937.

9.- (a) Kovvuri, V. R. R.; Xue, H.; Romo, D. Org. Lett. 2020, 22, 1407-1413. (b) Garlets, Z. J.; Silvi, M.; Wolfe, J. P. Org. Lett. 2016, 18, 2331-2334. (c) Mailyan, A. K.; Young, K.; Chen, J. L.; Reid, B. T.; Zakarian, A. Org. Lett. 2016, 18, 55325535. (d) Tian, M.; Yan, M.; Baran, P. S. J. Am. Chem. Soc. 2016, 138, 14234-14237. (e) Lemrová, B.; Soural, M. Eur. J. Org. Chem. 2015, 1869-1886.

10.- (a) Abdjul, D. B.; Yamazaki, H.; Kanno, S.; Takahashi, O.; Kirikoshi, R.; Ukai, K.; Namikoshi, M. J. Nat. Prod. 2016, 79, 1149-1154. (b) Ma, Y.; O’Doherty, G. A. Org. Lett. 2015, 17, 5280-5283. (c) Zaed, A. M.; Sutherland, A. Org. Bioorg. Chem. 2010, 8, 4394-4399. (d) Meragelman, K. M.; McKee; T. C.; McMahon, J. B. J. Nat. Prod. 2004, 67, 1165-1167.

11.- (a) Fleming, J. J.; McReynolds, M. D.; Du Bois, J. J. Am. Chem. Soc. 2007, 129, 9964-9975. (b) Fleming, J. J.; Du Bois, J. J. Am. Chem. Soc. 2006, 128, 3926-3927.

12.- Jadhav, P. K.; Woerner, F. J.; Lam, P. Y. S; Hodge, C. N.; Eyermann, C. J.; Man, H.-W.; Daneker, W. F.; Bacheler, L. T.; Rayner, M. M.; Meek, J. L.; Erickson-Viitanen, S.; Jackson, D. A.; Calabrese, J. C.; Schadt, M.; Chang, C.-H. J. Med. Chem. 1998, 41, 1446-1455.

13.- For review, see: Suárez, E.; Rodríguez, M. S. $\beta$-Fragmentation of Alkoxyl Radicals: Synthetic Applications. In Radicals in Organic Synthesis; Renaud, P., Sibi, M. P.; Eds., Wiley-VCH: Weinheim, 2001, Vol. 2, pp 440-454. For selected papers on $\beta$-Fragmentation of the research group, see: (a) Hernández-Guerra, D.; Rodríguez, M.S.; Suárez, E. Eur. J. Org. Chem. 2014, 23, 5033-5038; (b) Francisco, C.G.; González, C. C.; Kennedy, A. R.; Paz, N. R.; Suárez, E. Chem. Eur. J. 2008, 14, 6704-6712; (c) Francisco, C.G.; González, C. C.; Kennedy, A. R.; Paz, N. R.; Suárez, E. Tetrahedron Lett. 2006, 47, 35-38; (d) González, C. C.; Kennedy, A. R.; León, E. I.; Riesco-Fagundo, C.; Suárez, E. Chem. Eur. J. 2003, 9, 5800-5809; (e) Francisco, C. G.; González, C. C.; Kennedy, A. R.; Paz, N. R.; Suárez, E. Org. Lett. 2003, 5, 4171-4173.

14.- Compain, P. Chem. Rec. 2020, 20, 10-22.

15.- (a) Francisco, C.G.; Freire R.; González, C.C.; Suárez, E. Tetrahedron:Asymmetry 1997, 8, 1971-1974; (b) Santana, A. G.; Paz, N. R.; Francisco, C. G.; Suárez, E; González, C. C. J. Org. Chem. 2013, 78, 7527-7543. (c) Paz, N. R.; Santana, A. G.; Francisco, C. G., Suárez, E.; González C. C. Org. Lett. 2012, 14, 3388-3391. (d) André-Joyaux, E.; Santana, A. G.; González, C. C. J. Org. Chem. 2019, 84, 506-515.

16.- Santana, A. G.; Francisco, C. G.; Suárez, E; González, C. C. J. Org. Chem. 2010, 75, 5371-5374.

17.- Kwon, K.-H.; Edwards, A. V.; Yang, M.; Looper, R. E. Tetrahedron 2017, 73, 6067-6079.

18.- (a) Le Merrer, Y.; Gauzy, L.; Gravier-Pelletier, C.; Depezay, J.-C. Bioorg. Med. Chem. 2000, 8, 307-320. (b) Le, V.-D.; Wong, C.-H. J. Org. Chem. 2000, 65, 2399-2409. (c) Jeong, J.-H.; Murray, B. W.; Takayama, S.; Wong, C.-H. J. Am. Chem. Soc. 1996, 118, 4227-4234.

19.- Feichtinger, K.; Sings, H. L.; Baker, T. J.; Matthews, K.; Goodman, M. J. Org. Chem. 1998, 63, 8432-8439.

20.- Herrera-González, I.; Sánchez-Fernández, E. M.; Sau, A.; Nativi, C.; García Fernández, J. M.; Galán, M. C.; Ortiz Mellet, C. J. Org. Chem. 2020, 85, 5038-5047. 